\title{
Neurotoxic Lesions of the Lateral Nucleus of the Amygdala Decrease Conditioned Fear But Not Unconditioned Fear of a Predator Odor: Comparison with Electrolytic Lesions
}

\author{
Karin J. Wallace and Jeffrey B. Rosen \\ Department of Psychology and Neuroscience Program, University of Delaware, Newark, Delaware 19716
}

Considerable evidence suggests that the lateral (LA) and basal (BA) nuclei of the amygdala are sites of plasticity and storage of emotional memory. Recent arguments, however, have seriously challenged this view, suggesting that the effects of amygdala lesions are attributable to interference with performance of fear behavior and not learning and memory. One way to address this controversy is to measure the same behavioral response during both conditioned and unconditioned fear. This is done in the present study by measuring fear-related freezing behavior after electrolytic and neurotoxic lesions of the LA or LA/BA nuclei in rats in a contextual fear conditioning paradigm and unconditioned fear to a predator odor. Electrolytic LA lesions attenuated post-shock freezing, retention test freezing, and freezing to the predator odor trimethylthiazoline (TMT). In contrast, excitotoxic NMDA lesions of the LA had no effect on post-shock freezing

Much progress in understanding the neuroanatomy of fear has resulted from using simple well defined paradigms that measure species-typical defense responses primarily in rodents (e.g., freezing, fear-potentiated startle, and autonomic changes) during both cue-specific (e.g., light and tone) and contextual classical fear conditioning (i.e., environment without specific cue). It is generally accepted that the basolateral complex of the amygdala (BLC), consisting primarily of the lateral (LA) and basal (BA) amygdaloid nuclei, plays a major role in receiving and integrating conditioned and unconditioned stimuli for fear conditioning (Davis, 1997; Fanselow and LeDoux, 1999; Maren, 1999a; LeDoux, 2000). Indeed, electrolytic or neurotoxic damage to the LA or BLC block the learning of conditioned fear (LeDoux et al., 1990a; Kim et al., 1993; Campeau and Davis, 1995; Maren et al., 1996a; Cousens and Otto, 1998). This evidence, together with electrophysiological data demonstrating neural activity changes during fear conditioning (Quirk et al., 1995; Rogan et al., 1997; Collins and Pare, 2000; Maren, 2000; Pare and Collins, 2000) and blockade of fear learning after pharmacological manipulation in the amygdala (Miserendino et al., 1990; Campeau et al., 1992; Maren et al., 1996b; Muller et al., 1997; Wilensky et al., 2000), suggest that the BLC is a site of plasticity and storage of emotional information (Fanselow and LeDoux, 1999).

Received Oct. 23, 2000; revised Feb. 27, 2001; accepted Feb. 27, 2001.

This study was supported by National Science Foundation Grant IBN-9904623 (J.B.R.).

Correspondence should be addressed to Dr. Jeffrey B. Rosen, Department of Psychology, University of Delaware, 220 Wolf Hall, Newark, DE 19716. E-mail: jrosen@udel.edu.

Copyright (C) 2001 Society for Neuroscience 0270-6474/01/213619-09\$15.00/0 but significantly attenuated retention test freezing. Furthermore, excitotoxic LA lesions did not diminish freezing to TMT. Larger excitotoxic lesions that included the BA significantly reduced freezing in both the post-shock and retention tests but did not appreciably decrease freezing to TMT. The results suggest that the LA is important for memory of learned fear but not for generation of freezing behavior. In addition, the BA plays a role in freezing in conditioned fear situations but not in unconditioned fear. The studies suggest that the LA and BA play different roles in fear conditioning, but neither of them has a significant role in unconditioned freezing to a predator odor.

Key words: fear conditioning; context conditioning; amygdala; basolateral nucleus of the amygdala; lateral nucleus of the amygdala; basal nucleus of the amygdala; unconditioned fear; fox odor; freezing

Recently, serious challenge to the view that the BLC is a locus of conditioned fear has emerged (Cahill et al., 1999; Vazdarjanova, 2000). One major argument for this challenge is that there is little direct evidence that distinguishes between the effects of BLC lesions on memory versus performance. Thus, reduction in behavioral measures of conditioned fear (primarily freezing and fear-potentiated startle) in BLC-lesioned animals may be attributable to a reduction of learning or may simply be a consequence of an inability of the lesioned animal to perform the necessary behavior. Cahill et al. (1999) suggest that, until it is shown that BLC lesions block conditioned but not unconditioned fear behavior, it is premature to conclude that the BLC is necessary for learning and memory of conditioned fear.

The present study directly addresses this issue. Presentation of a predator odor, trimethylthiazoline [(TMT) originally isolated from fox feces] has been shown to elicit a number of fearassociated responses in rats (Cattarelli and Chanel, 1979; VernetMaury et al., 1984, 1992; Heale et al., 1994; Hotsenpillar and Williams, 1997; Morrow et al., 2000a,b; Wallace and Rosen, 2000). Most relevant to our application is the demonstration that rats reliably display robust unconditioned freezing behavior to TMT (Wallace and Rosen, 2000). Thus, it is possible to measure the same fear-related behavior, freezing, during acquisition and retrieval of conditioned fear and during the presence of an ecologically relevant unconditioned fear stimulus in the same amygdala-lesioned rats. To determine whether both types of fear are disrupted by specific amygdala damage, the effects of both electrolytic and neurotoxic lesions of the LA or combined LA and BA lesions on conditioned and unconditioned fear-related freezing were analyzed. 


\begin{tabular}{llllllll}
\hline \multicolumn{2}{l}{ Table 1. Electrolytic lesion parameters } & & & \\
Squad & $\begin{array}{l}\text { Location } \\
\text { of lesion }\end{array}$ & Current & Time & $\begin{array}{l}\text { Posterior } \\
\text { coordinate }\end{array}$ & $\begin{array}{l}\text { Lateral } \\
\text { coordinate }\end{array}$ & $\begin{array}{l}\text { Ventral } \\
\text { coordinate }\end{array}$ & $\begin{array}{l}\text { Animals per } \\
\text { group }\end{array}$ \\
\hline \multirow{4}{*}{1} & Anterior & $0.1 \mathrm{~mA}$ & $90 \mathrm{sec}$ & 2.6 & 5.0 & 7.8 & \\
& Mid & $0.1 \mathrm{~mA}$ & $90 \mathrm{sec}$ & 3.2 & 5.0 & 7.8 & LA, 1 \\
& Posterior & $0.1 \mathrm{~mA}$ & $90 \mathrm{sec}$ & 3.7 & 5.1 & 7.7 & LA/ASTR, 2 \\
& Anterior & $0.1 \mathrm{~mA}$ & $30 \mathrm{sec}$ & 2.6 & 5.0 & 7.8 & \\
& Mid & $0.1 \mathrm{~mA}$ & $30 \mathrm{sec}$ & 3.2 & 5.0 & 7.8 & LA, 5 \\
& Posterior & $0.1 \mathrm{~mA}$ & $30 \mathrm{sec}$ & 3.7 & 5.1 & 7.7 & LA/ASTR, 0 \\
& Anterior & $0.1 \mathrm{~mA}$ & $30 \mathrm{sec}$ & 2.6 & 4.9 & 7.8 & \\
& Mid & $0.1 \mathrm{~mA}$ & $45 \mathrm{sec}$ & 3.2 & 4.9 & 7.8 & LA, 0 \\
& Posterior & $0.1 \mathrm{~mA}$ & $45 \mathrm{sec}$ & 3.9 & 5.1 & 7.8 & LA/ASTR, 6 \\
& Anterior & $0.1 \mathrm{~mA}$ & $30 \mathrm{sec}$ & 2.6 & 4.9 & 7.8 & LA, 8 \\
& Mid & $0.1 \mathrm{~mA}$ & $45 \mathrm{sec}$ & 3.2 & 4.9 & 7.8 & LA/ASTR, 1 \\
\hline
\end{tabular}

Location of lesion refers to lesion sites at three rostrocaudal levels of the amygdala. Time is the duration of current given at each lesion site. All coordinates are millimeters from bregma. The number of animals per squad included in the LA or LA/ASTR groups are given in the Animals per group column.

\section{MATERIALS AND METHODS}

\section{Subjects}

Male Sprague Dawley rats (Harlan Sprague Dawley, Indianapolis, IN) weighing 250-300 gm at surgery were used in these experiments. They were maintained on a $12 \mathrm{hr}$ light/dark cycle, and food and water were available ad libitum. All experiments were approved by the University of Delaware Institutional Animal Care and Use Committee.

\section{Apparatus}

During fear conditioning, rats were placed in a Plexiglas cylindrical chamber $(8.6 \mathrm{~cm}$ diameter, $20 \mathrm{~cm}$ long; SR-Lab animal enclosure; San Diego Instruments, San Diego, CA). Plexiglas doors dropped into slots at each end of the cylinder kept the rat in the chamber. The rat was confined but not restrained and could move and turn around in the chamber, but when not turning faced one of the two doors. The cylinder was mounted on a Plexiglas platform inside a cabinet of particleboard covered with Formica $(30 \times 30 \times 60 \mathrm{~cm}$; S-R chambers; San Diego Instruments). A grid floor (San Diego Instruments) consisting of seven parallel stainless steel rods, each measuring $4 \mathrm{~mm}$ in diameter and spaced $1.3 \mathrm{~cm}$ apart (center to center), was placed on the floor of the cylinder and was attached to a scrambled shocker. A $25 \mathrm{~W}$ light bulb located in the roof of the cabinet was on at all times, and a fan in the cabinet provided a background noise of $70 \mathrm{~dB}$.

The same Plexiglas cylinders without the grid floors were used in the predator odor experiment but were placed in a fume hood rather than the Formica-particleboard chambers. The fume hood was used to prevent the volatile odorant TMT from spreading into the experimental room. TMT was presented to the animal by pipetting it onto a piece of Kimwipe that was taped to the inside of each door. In both fear conditioning and presentation of the predator odor, the chamber was cleaned with $5 \%$ ammonium hydroxide after running each animal. In the TMT experiments, residual odor was also allowed to dissipate in the fume hood before another animal was brought into the experimental room and placed in the chamber.

\section{Procedure}

Electrolytic lesions. Rats were anesthetized with a ketamine $(100 \mathrm{mg} / \mathrm{kg}$, i.p.) and xylazine $(6.7 \mathrm{mg} / \mathrm{kg}$, i.p.) solution for surgery. Bilateral lesions of the LA were made in three locations along the rostrocaudal extent of the nucleus $\sim 0.6 \mu \mathrm{m}$ apart. Lesions were made in four squads of rats with slightly different coordinates and duration of current (Table 1). Stainless steel electrodes of $250 \mu \mathrm{m}$ diameter, insulated except for $500 \mu \mathrm{m}$ at the tip, were used (model NE-300; Rhodes Medical Instruments, Woodland Hills, CA). Lesions were generated by passing a $0.1 \mathrm{~mA}$ anodal current through the electrode tip. The cathode was attached to the rat's foot with an alligator clip. Rats were allowed 7-10 d to recover, during which time they were also handled.

NMDA lesions. Rats were anesthetized as described for electrolytic lesions. Bilateral lesions of the LA were made by a single injection of
NMDA (Sigma, St. Louis, MO) in each amygdala. Lesions were made in three squads of animals using different amounts of NMDA to obtain different sizes of damage. The needle of a $1.0 \mu$ l Hamilton syringe (Hamilton Company, Reno, NV) was lowered to the target site (from bregma: posterior, $3.3 \mathrm{~mm}$; lateral, $\pm 4.9 \mathrm{~mm}$; and ventral, $7.8 \mathrm{~mm}$ ) and left in place for $2 \mathrm{~min}$ before injection. NMDA $(20 \mathrm{mg} / \mathrm{ml})$ was then infused at $0.05 \mu \mathrm{l} / \mathrm{min}$, for a total injection of $0.1,0.15$, or $0.2 \mu \mathrm{l}$. After infusion, the Hamilton syringe was left in place for an additional $5 \mathrm{~min}$ before removal, and the same procedure was followed for the other amygdala. Rats were allowed 7-10 d to recover, during which time they were also handled.

\section{Behavior}

Contextual fear conditioning. The simplest version of contextual fear conditioning was used in these experiments in which a rat receives a foot shock after being placed in a novel environment (Fanselow, 2000). Rats were placed in the chamber for $3 \mathrm{~min}$ before a $1.5 \mathrm{~mA}, 1 \mathrm{sec}$ foot shock. Freezing was measured for $4 \mathrm{~min}$ immediately after the foot shock (post-shock period). Freezing was defined as a characteristic crouch position with cessation of all movement except that associated with breathing (Blanchard and Blanchard, 1969). Freezing was measured as a sample of freezing or not freezing every $10 \mathrm{sec}$, for a total of 25 observations. The number of observations of freezing was divided by 25 and then multiplied by 100 to obtain a percent of time spent freezing.

A retention test of fear conditioning was conducted $24 \mathrm{hr}$ after the foot shock by placing the animals back into the same chamber and recording freezing for $4 \mathrm{~min}$ as described above. In both the post-shock and the retention tests, the observer was blind to the condition (lesion group of each rat). Freezing data were statistically analyzed with a mixed-model ANOVA (lesion group as a between-subjects measure and freezing tests as a within-subjects measure), followed by a Scheffe's $S$ post hoc test. $\alpha$ values were set at $p<0.05$.

Presentation of predator odor. After contextual fear conditioning, rats freezing behavior in the cylinders was extinguished in the fume hood before presentation of TMT until they froze $<20 \%$ of the time (no odor, but a Kimwipe on the door). This required four to six $10 \mathrm{~min}$ sessions in the chamber once or twice per day, before being tested for freezing to TMT. On the testing day, rats were placed in the chamber for a $3 \mathrm{~min}$ acclimation period, after which the odorless doors were exchanged with doors containing $150 \mathrm{nmol}$ of TMT $(19.4 \mu \mathrm{l})$ on each door. Freezing was recorded for the following $11 \mathrm{~min}$ and was scored as a percent of time spent freezing as described for context conditioning. This amount of TMT was reported previously to elicit freezing $\sim 75-80 \%$ of the time (Wallace and Rosen, 2000) and at similar levels as found after a $1 \mathrm{sec}, 1.5$ $\mathrm{mA}$ foot shock in normal rats (Malkani and Rosen, 2000; Thompson and Rosen, 2000).

\section{Histology}

After the conclusion of each experiment, rats were overdosed with sodium pentobarbital and perfused transcardially with $0.1 \mathrm{M}$ PBS, fol- 
lowed by $4 \%$ formaldehyde in $0.1 \mathrm{M}$ PBS. Brains were removed, postfixed, and cryoprotected (4\% formaldehyde, $30 \%$ sucrose, and $0.1 \mathrm{M}$ PBS) for 4-10 d. Brains were frozen and sliced (Jung CM3000 cryostat; Leica, Deerfield, IL) at $40 \mu \mathrm{m}$, mounted onto microscope slides (Micro Slides, Selected, Precleaned Superfrost Plus; VWR Scientific, West Chester, $\mathrm{PA})$, and stained with cresyl violet to determine lesion locations.

Lesions were located using a light microscope and mapped onto computerized coronal brain drawings from the Paxinos and Watson rat brain atlas (1998) using NIH Image 1.59 on a 7600/132 Power Macintosh (Apple Computers, Cupertino, CA). This was done for six brain sections in each animal, covering the anteroposterior extent of the amygdala. Analysis of lesion size in four nuclei [LA, BA, amygdalostriatal transition region (ASTR), and caudate putamen $(\mathrm{CPu})]$ was conducted. For analysis of each lesion, the area of each nucleus and the area of the lesion in the nucleus were measured. Only the ventral half of the $\mathrm{CPu}$ was used as the area of the nucleus measured because damage was confined to the ventral $\mathrm{CPu}$. A percent of each nucleus lesioned was calculated by dividing the area of the lesion in the nucleus by the total area of the nucleus and multiplying by 100 . These scores from the six drawings were then averaged to get a total percent of the nucleus damaged.

\section{RESULTS}

\section{Electrolytic lesions}

\section{Histology}

Subjects were divided into three groups based on histology (LA, LA/ASTR, and no lesion). Most of the LA rats were from squads 2 and 4, whereas ASTR rats were mostly from squad 3 (Table 1). Rats were included in the LA group if the lesion was centered in the LA with only minor damage to the surrounding nuclei. LA lesions were bilateral and always included the dorsolateral division of the LA. Rats with lesions of the LA that included extensive lesions of surrounding areas were excluded. Based on these criteria, 14 rats were included in the LA lesion group (Figs. $1 A, 2)$. The mean \pm SEM percentage of the LA destroyed was $56 \pm 4 \%$. In some rats, there was also slight damage to the dorsal endopiriform nucleus (11 $\pm 2 \%$ of the nucleus), the BA $(7 \pm 2 \%$ of the nucleus), the ASTR ( $9 \pm 1 \%$ of the region), and/or the $\mathrm{CPu}(9 \pm 2 \%$ of the ventral half of the nucleus). The LA/ASTR group $(n=9)$ included animals with lesions of both the LA and the adjacent ASTR (Fig. 2). This group generally had smaller lesions of the LA (41 $\pm 7.5 \%$ of the nucleus) but larger lesions of the ASTR ( $32 \pm 4 \%$ of the region) compared with the LA group. One animal included in this group had very minor damage to the LA but significant damage to the ASTR (Fig. $1 B$ ). All rats in the LA/ASTR group also had some damage to the CPu (29 $\pm 3 \%$ of the ventral half of the nucleus). Damage to the LA was primarily concentrated in the dorsolateral portion, and damage to the ASTR tended to be located laterally and close to the LA. The final no-lesion group consisted of animals with sham lesions of the amygdala $(n=4)$ and no surgery $(n=11)$. The behavior of these two subgroups did not differ and so were combined for analysis. Fifteen rats were not included in the analysis because their lesions were asymmetrical, unilateral, or not in the LA and could not be placed in a group. One of these rats was excluded from analysis because the lesion of the LA was small (24\%), although it did have attenuated freezing.

\section{Effects of electrolytic lesions on contextually conditioned freezing}

Once three groups were formed based on the lesions, context conditioning was analyzed using a 3 (groups LA, LA/ASTR, and no lesion; between measure) $\times 2$ (post-shock vs retention; within measure) mixed-model ANOVA. The data are presented in graphic form in Figure 3. There was an overall difference between the groups during context conditioning ${\left(F_{(2,31)}=53.1 ; p<\right.}$
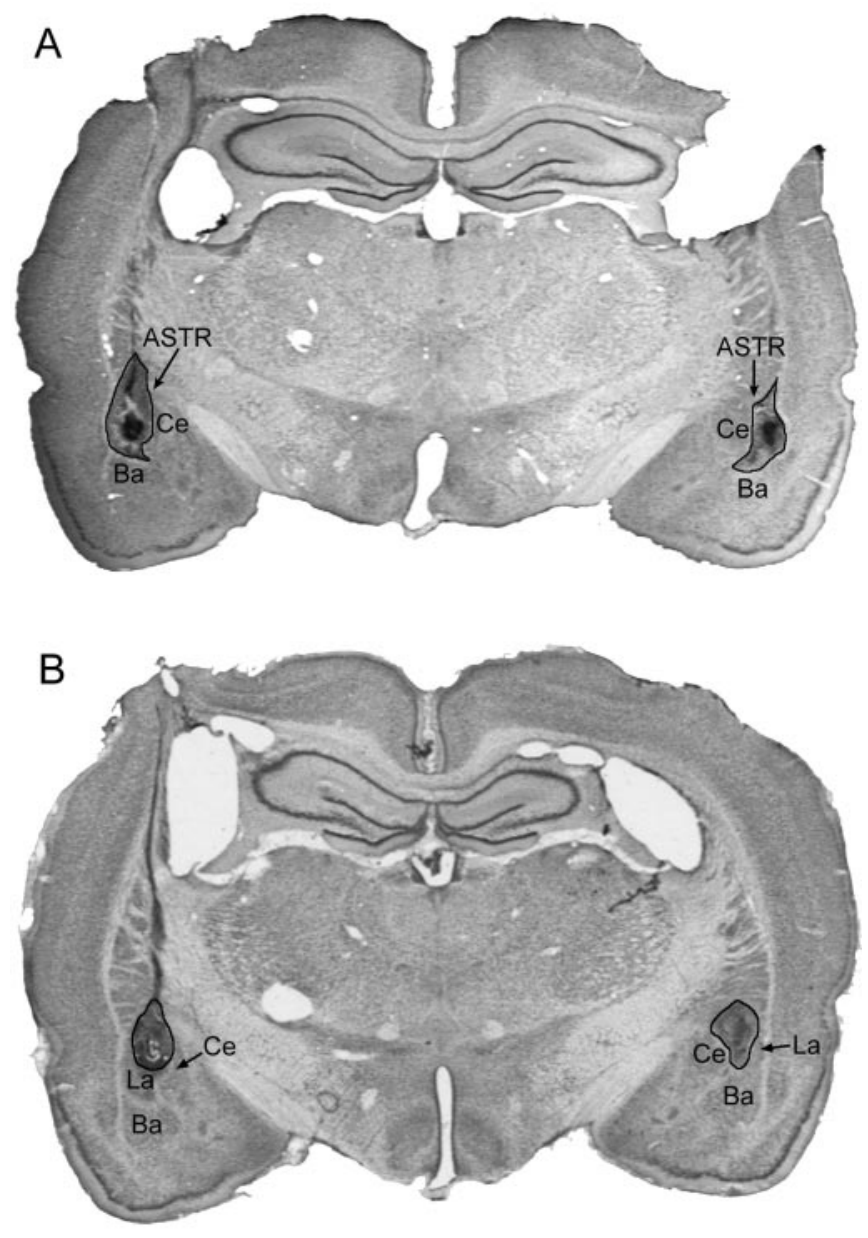

Figure 1. Coronal brain sections of electrolytic lesions from the LA group $(A)$ and LA/ASTR group $(B)$. The lesions are outlined in black. In $A$, the LA lesions had only slight damage of the ASTR, basal nucleus of the amygdala, and central nucleus of the amygdala. The lesions of the rat shown in $B$ were primarily located in the ASTR, sparing much of the LA. This rat was unique because other rats in the LA/ASTR group had lesions of both the LA and ASTR. Behaviorally, attentuation of freezing in this rat was similar to the rest of the group. $B a$, Basal nucleus of the amygdala; $C e$, central nucleus of the amygdala; $L a$, lateral nucleus of the amygdala.

0.0001). Scheffe's $S$ post hoc test demonstrated that rats with lesions of the LA and LA/ASTR froze significantly less than rats in the no-lesion group. A difference in freezing between the LA/ASTR and the LA-lesioned rats failed to reach statistical significance $(p<0.063)$. There was also an overall difference between the post-shock and the retention tests $\left(F_{(1,31)}=15.2 ; p<\right.$ $0.0005)$. However, there was no interaction effect between the groups and their levels of freezing during the post-shock and retention tests $\left(F_{(2,31)}=0.23\right)$, indicating that the effects of LA and LA/STR lesions were an overall effect on a reduction in freezing in both the post-shock and retention tests compared with the no-lesion group.

These results of a significant attenuation of freezing complements a previous study of electrolytic lesions of the LA demonstrating deficits in cue-specific conditioned freezing during a retention test (LeDoux et al., 1990a). Post-shock freezing was not analyzed. Together, the results of the two studies indicate that the LA plays a role in both cue-specific and contextual fear conditioning paradigms. In addition, our study indicates that larger 


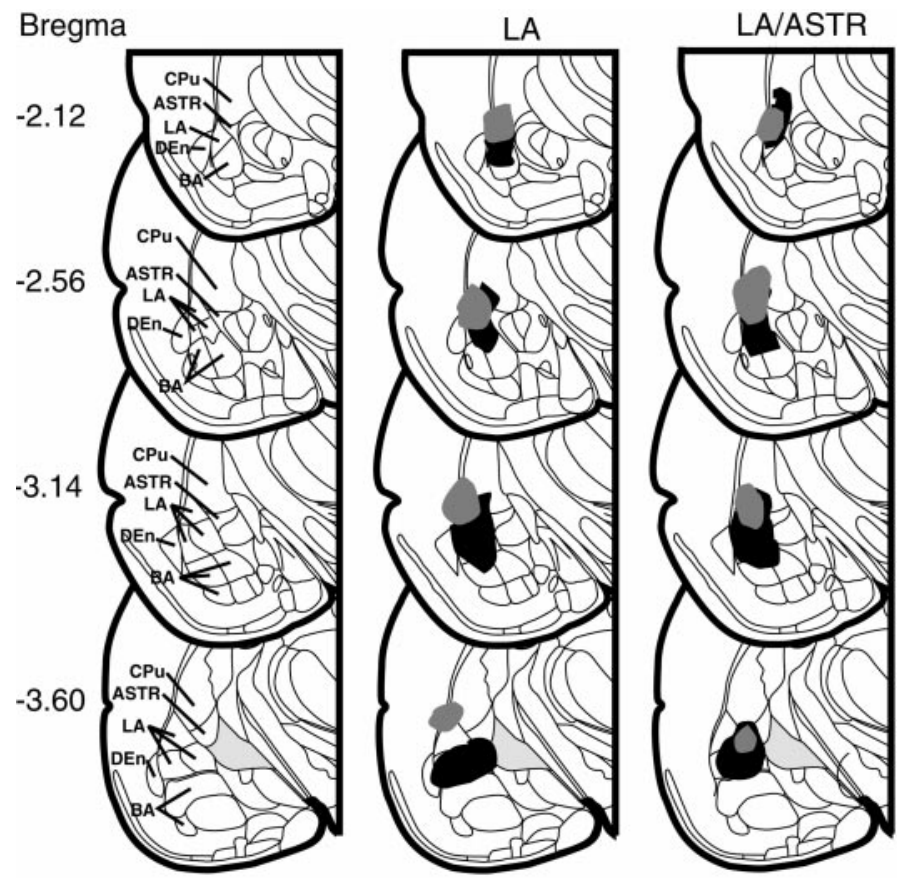

Figure 2. Schematic representations of largest and smallest electrolytic lesions in the amygdala at four rostral to caudal coronal levels (numbers indicate millimeters posterior from bregma). The sections are of the left ventral quadrants of coronal drawings containing the amygdala from the atlas of Paxinos and Watson (1998). Relevant structures are labeled in the left row of drawings. Lesions of the LA and LA/ASTR are in the middle and right rows, respectively. The gray areas represent the animals with smallest lesion and the black areas represent the largest lesion of each group. DEn, Dorsal endopiriform nucleus.

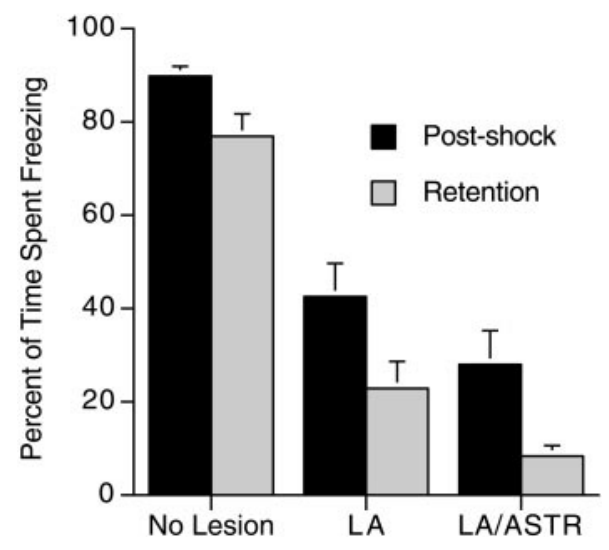

Figure 3. Effects of electrolytic lesions on short-term and long-term memory of conditioned fear as measured by the percent of time spent freezing in the post-shock period and retention test, respectively. Freezing in the post-shock period was significantly more than during the retention test for all groups. Overall freezing (both post-shock and retention) in the LA and LA/ASTR groups was significantly less that the no-lesion group. There was no interaction effect.

lesions that contain both the LA and ASTR, or even lesions of the ASTR alone (Fig. 1B), contribute only a slight nonsignificant additive effect to lesions confined to the LA. Although the present results and those of LeDoux et al. (1990a) seem to support a role of the LA in fear conditioning, a decrease of freezing in both the post-shock and retention test without a differential effect on these tests compared with the no-lesion group confounds this interpretation. An alternative interpreta-

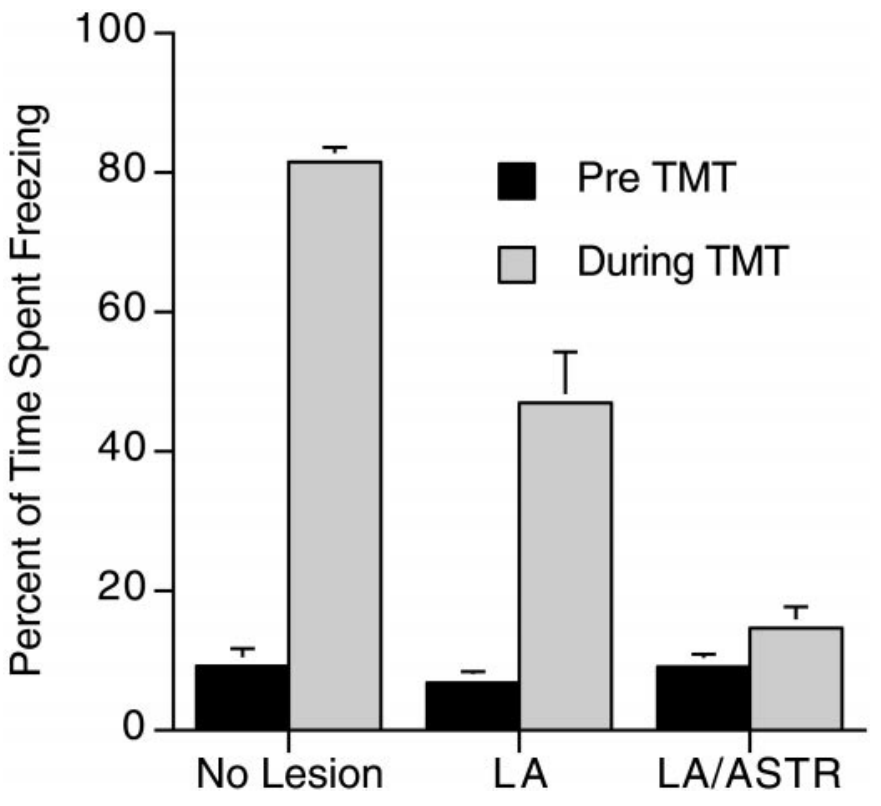

Figure 4. Percentage of time rats with electrolytic lesions of the amygdala spent freezing to TMT. Freezing before TMT presentation (Pre $T M T)$ did not differ between the groups. The three groups differed from each other on the percentage of freezing during TMT. Only the LA/ ASTR lesions completely blocked freezing (there was no significant difference in freezing before and during TMT).

tion is that the LA simply reduced the ability of the animals to freeze without affecting learning (for a detailed argument of this interpretation, see Cahill et al., 1999). This problem is addressed in the section on the effects of NMDA lesions on fear conditioning. Those data are presented after presentation of the effects of electrolytic lesions of the LA and LA/ASTR on unconditioned freezing to a predator odor.

Effects of electrolytic lesions on unconditioned freezing to TMT On the day of TMT testing, rats froze on average $<10 \%$ of the 3 min period before TMT was introduced into the chamber, and there was no difference between the groups (mean \pm SEM; LA, $6.8 \pm 1.6 \%$; LA/ASTR, $9.1 \pm 1.8 \%$; no lesion, $9.2 \pm 2.5 \%$ ). Differences in freezing behavior before and during TMT presentation between the groups were analyzed using a 3 (groups LA, LA/ASTR, and no lesion; between measure) $\times 2$ (pre-TMT vs during TMT; within measure) mixed-model ANOVA. The data are presented in graphic form in Figure 4. There was less freezing before TMT than during TMT presentation $\left(F_{(1,2)}=149.4 ; p<\right.$ $0.0001)$ and an overall difference in freezing between the LA, LA/ASTR, and no-lesion groups $\left(F_{(2,32)}=27.2 ; p<0.0001\right)$. A Scheffe's $S$ post hoc test showed that all groups were significantly different from each other, in which the no-lesion group had the highest and the LA/ASTR group had the lowest levels of freezing. In addition, there was an interaction between the groups freezing in the pre-TMT and during TMT periods $\left(F_{(2,32)}=33.0\right.$; $p<0.0001)$. This is attributable to a difference in freezing between pre-TMT and during TMT for the LA lesion and the no-lesion groups but not the LA/ASTR group. A paired $t$ test on the pre-TMT and during TMT freezing data in the LA/ASTR group was not significant $\left(t_{(10)}=1.81 ; p>0.1\right)$.

The data demonstrate that rats with lesions of the LA froze less than rats without lesions, although the lesions did not completely block freezing. In contrast, freezing was blocked with combined 

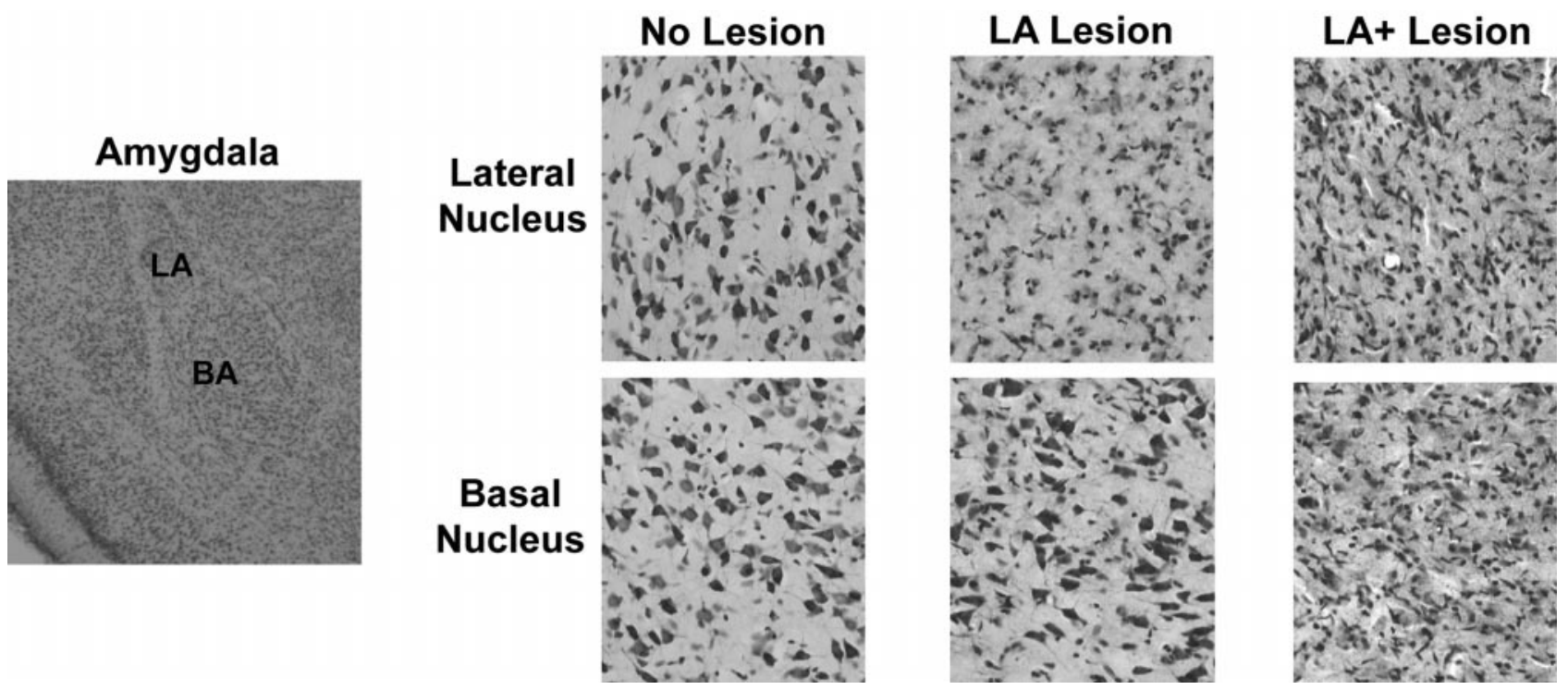

Figure 5. Representative NMDA lesions in the amygdala. On the left is a low-magnification cresyl violet-stained coronal section of the amygdala. On the right are magnified regions $(50 \times)$ of the lateral and basal nuclei of a single coronal section from each of the three experimental groups. The No Lesion specimen demonstrates normal cell morphology in lateral and basal nuclei. In the LA Lesion samples, gliosis dominates the lateral nucleus, but normal neuronal cell morphology is found the basal nucleus. The $L A+$ Lesion samples have only gliosis in both the lateral and basal nuclei.

lesions of the LA/ASTR and in one rat with lesions of the ASTR, suggesting that either the ASTR or some combination of the LA and ASTR is important for contextually conditioned freezing and freezing to TMT. The ASTR is a small poorly characterized region between the LA ventrally and laterally, the central nucleus medially, and the caudate putamen dorsally, but it is usually included as part of the central nucleus of the amygdala because of cell morphology (McDonald, 1998). However, LeDoux et al.(1990b) have shown that thalamic areas, including the posterior intralaminar nucleus and the medial posterior complex, which project to the LA, also project to the ASTR. Shi and Cassell (1999) reported recently that fibers from the perirhinal cortex, a region that projects to the LA, also terminate in the ASTR. Therefore, it is possible that the LA/ASTR lesions eliminated more of the afferent sensory fields important for conditioned and unconditioned freezing than did LA lesions alone. Nevertheless, what remains unresolved is the question of whether the effects produced by electrolytic lesions are attributable to cells in the LA and ASTR or to fibers passing through these regions. The results from excitotoxic NMDA lesions presented below address this question.

\section{NMDA lesions \\ Histology}

Groups were determined by histological analysis of the area destroyed. Of the 67 rats infused with NMDA, only 25 had sufficient damage to assign them to a group. Rats with lesions on only one side of the brain were excluded because the interest was in the overall effects of LA lesions on freezing. In addition, some animals were excluded based on lesion placement outside of the amygdala. The remaining animals were divided into two lesion groups (Figs. 5, 6) and one no-lesion group based on location and extent of the lesions. One lesion group was formed with lesions in the LA $(n=8)$. Rats in this group had $48.1 \pm 6.2 \%$ (mean \pm SEM) of the LA destroyed bilaterally, with the entire dorsolateral division of the LA destroyed in all animals except for two, in which the most dorsal tip of the nucleus was left undamaged.
Animals with significant damage to the basal nucleus were not included in the LA group [only $3.9 \pm 1.1 \%$ (mean \pm SEM) of BA was lesioned]. Lesions frequently extended somewhat beyond the LA laterally and dorsally and included parts of the dorsal endopiriform nucleus $[32.1 \pm 5.8 \%$ (mean \pm SEM) of the nucleus
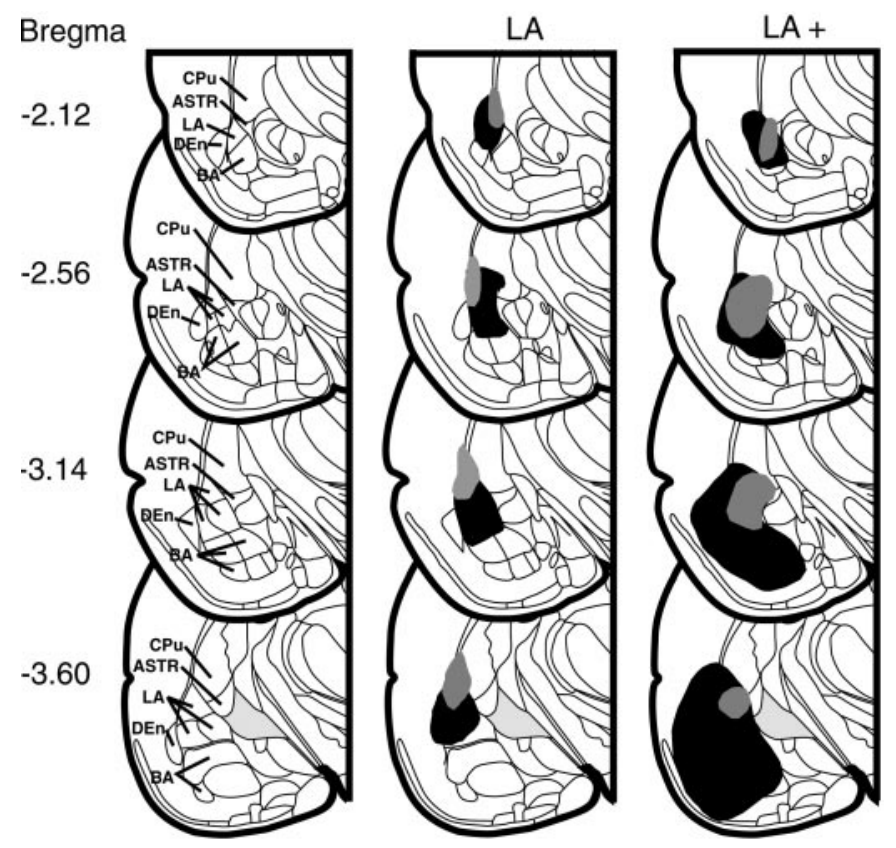

Figure 6. Schematic representations of largest and smallest NMDA lesions in the amygdala at four rostral to caudal coronal levels (numbers indicate millimeters posterior from bregma). The sections are of the left ventral quadrants of coronal drawings containing the amygdala from the atlas of Paxinos and Watson (1998). Relevant structures are labeled in the left row. Lesions of the LA and LA+ groups are in the middle and right rows, respectively. The gray areas represent the animals with smallest lesion and the black areas represent the largest lesion of each group. DEn, Dorsal endopiriform nucleus. 


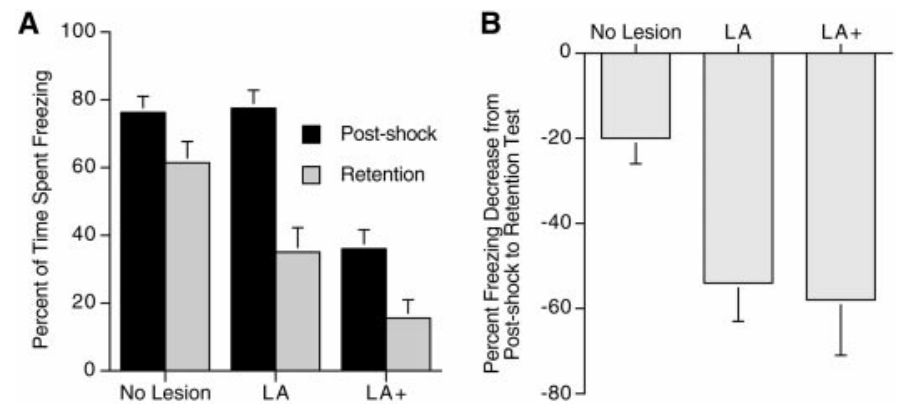

Figure 7. NMDA lesion of the LA and LA+ on conditioned fear. $A$, Effects of NMDA lesions on short-term and long-term contextually conditioned fear as measured by the percentage of time spent freezing in the post-shock period and retention test, respectively. Freezing in the postshock period was significantly more than during the retention test for all groups. The LA+ group had significantly less overall freezing than the no-lesion and LA groups. The post-shock freezing of the no-lesion and LA groups did not differ; however, there was significantly less freezing during the retention test in the LA group compared with the no-lesion group. $B$, The percentage of decrease in freezing in the retention test compared with the post-shock period. Both the LA and LA+ groups had a significantly greater percentage decrease of freezing in the retention test from the post-shock period than the no-lesion group.

was damaged], the $\mathrm{CPu}(19.4 \pm 5.4 \%$ was damaged $)$, and/or the $\operatorname{ASTR}(17.0 \pm 4.1 \%$ was damaged $)$. The central nucleus of the amygdala was not damaged. Another group [LA lesion plus damage to surrounding areas (LA+); $n=17$ ] (Figs. 5, 6) consisted of larger lesions of the LA (78.1 $\pm 4.9 \%$ was damaged), as well as more damage to other nuclei: the BA $(34.4 \pm 6.1 \%$ was damaged), ASTR (63.2 $\pm 4.0 \%$ was damaged), caudate putamen (37 $\pm 2.4 \%$ was damaged), and piriform cortex (specific amount of damage was not determined). Again, the central nucleus of the amygdala was not damaged. A final group did not have a visible lesion on either side of the brain (no lesion; $n=8$ ). Some of the cases in the no-lesion group had cannula tracks that were discernable near the caudal end of the amygdala but did not appear to have a lesion surrounding the track. An additional group was added to the analysis for behavioral comparison that did not have surgery $(n=9)$. Because their amount of conditioned freezing was no different from the no-lesion group they were included in the no-lesion group, which increased the total number of subjects to 17. Unfortunately, a separate group with NMDA lesions confined to the ASTR could not be formed.

\section{Effects of NMDA lesions on contextually conditioned freezing}

For statistical analysis of the effects of NMDA lesions on contextual fear conditioning, a 3 (groups LA, LA+, and no lesion; between measure) $\times 2$ (post-shock vs retention; within measure) mixed-model ANOVA was conducted. There was an overall difference between the groups $\left(F_{(2,39)}=22.8 ; p<0.0001\right)$. Scheffe's $S$ post hoc analysis revealed that the difference was attributable to a lower level of freezing in rats in the LA+ group compared with LA and no-lesion groups. This indicates that, overall, there was not an effect of NMDA lesions of the LA on freezing, but the larger lesions in the $\mathrm{LA}+$ animals produced significant decreases in freezing. There was also a significant overall post-shock versus retention difference $\left(F_{(1,2)}=48.8 ; p<0.0001\right)$ attributable to lower freezing scores on the retention test compared with freezing in the post-shock period. Most importantly, there was a significant interaction effect between the groups and testing pe$\operatorname{riod}\left(F_{(2,39)}=4.2 ; p<0.02\right)($ Fig. $7 A)$.

To further analyze this significant interaction, two additional analyses were performed. Post-shock freezing between the groups was compared using a one-way ANOVA, followed by a Scheffe's $S$ test. The significant difference in post-shock freezing $\left(F_{(2,39)}=\right.$ 20.0; $p<0.0001)$ was attributable to diminished freezing in the $\mathrm{LA}+$ groups compared with the no-lesion and LA groups. The analysis demonstrates that NMDA lesions confined to the LA had no detrimental effects on freezing behavior per se, whereas larger lesions did. Because the post-shock freezing levels were different between the groups, an analysis of differences in retention of conditioned fear had to be corrected for these differential levels in initial freezing. To standardize freezing scores across the groups, a percent decrease in freezing score in the retention test and the post-shock period was derived for each subject by the following formula: (retention test freezing/post-shock freezing $\times$ $100-100$. This results in a percent decrease in freezing in the retention test compared with the post-shock period (Fig. 7B). A one-way ANOVA $\left(F_{(2,37)}=4.80 ; p<0.01\right)$ followed by a Student-Newman-Keuls post hoc test was performed on these derived scores. There was a significant difference $(p<0.05)$ between the LA and no-lesion groups and between the LA+ and no-lesion groups but not between the LA and LA+ groups. The analysis indicates that both lesions confined to the LA and larger lesions that destroyed the LA plus considerable portions of the BA, endopiriform nucleus, and ASTR produced significant decrements in freezing during the retention test compared with the no-lesion group. Furthermore, when post-shock freezing in the two lesion groups was compared with the no-lesion group (Fig. $7 A$ ), only freezing of the LA + group, but not the LA group, was diminished. This indicates that NMDA lesions confined to the LA had no detrimental effects on freezing behavior per se but did have specific effects on long-term memory of fear. In addition, because post-shock freezing is a measure of short-term memory, the results suggest that short-term memory for fear conditioning occurs independent of the LA. Lesions also including the ASTR and BA did decrease post-shock freezing, and possibly short-term memory, but did not contribute more to the deficits in long-term memory than lesions confined to the LA (Fig. 7B).

\section{Effects of NMDA lesions on unconditioned freezing to TMT}

A one-way ANOVA was conducted to determine differences in freezing to TMT between the groups (LA, LA+, and no lesion). There was a significant difference in freezing $\left(F_{(2,39)}=8.2 ; p<\right.$ $0.001)$. A Scheffe's $S$ post hoc analysis revealed a statistical difference between the no-lesion group and the LA+ group but no difference between the no-lesion group and lesions of just the LA. However, although there was a statistical difference between these groups, the drop in the amount of freezing to TMT was small. The LA + group still froze $\sim 63 \%$ of the time compared with $79 \%$ for the no-lesion group and was not statistically different from the LA group freezing of $67 \%$ of the time (Fig. 8).

To ensure that the lack of a reduction in freezing to TMT in the NMDA lesion rats was not attributable to an effect of being tested after fear conditioning, two rats were tested for freezing to TMT before fear conditioning. These rats displayed the same reduction of fear conditioning freezing without an effect on freezing to TMT (73 and $67 \%$ of the time spent freezing to TMT).

\section{DISCUSSION}

The results of the present study demonstrate that electrolytic lesions of the LA or the LA/ASTR substantially diminish freezing in a contextually conditioned fear paradigm. These lesions also reduced unconditioned freezing to a predator odor. The data 


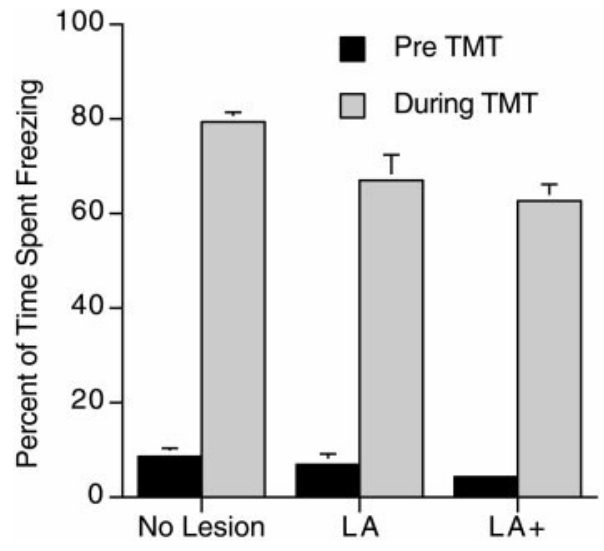

Figure 8. Percentage of time rats with NMDA lesions of the amygdala spent freezing to TMT. Freezing before TMT presentation did not differ between the groups. There was a slight but statistically significant decrease in freezing in the LA+ group compared with the no-lesion group.

tend to support Cahill et al.'s (1999) hypothesis that amygdala lesions interfere with freezing instead of learning and memory. In contrast, excitotoxic lesions of the LA had no effect on postshock- or predator odor-induced freezing but significantly reduced freezing during the retention test for contextually conditioned fear. These results suggest that the cells of the LA play a role in learning and memory of fear conditioning, whereas fibers passing through the LA and/or ASTR play a role in the expression of freezing behavior. Larger excitotoxic lesions that also included part of the BA and ASTR produced deficits in both shock-induced freezing and freezing during the retention test but had negligible effects on freezing to a predator odor. Therefore, contrary to the suggestion of Cahill et al. (1999), our results strongly indicate that BLC is not necessary for production of freezing behavior. The data also suggest the following: (1) the LA plays a specific role in long-term memory of fear, (2) the BA and ASTR are involved in both short- and long-term memory, and (3) the neuroanatomy of fear conditioning and unconditioned fear to a predator odor are different.

\section{Role of LA in fear conditioning}

Auditory, visual, and somatic information from the cortex and thalamus enters the amygdala primarily via the LA (Pitkanen et al., 1997; McDonald, 1998; Swanson and Petrovich, 1998). During fear conditioning, association between conditioned and unconditioned stimuli is thought to occur in the LA (Davis, 1997; Maren, 1999a; LeDoux, 2000). Electrolytic lesions confined to the LA block auditory cue-specific fear conditioning (LeDoux et al., 1990a), and electrophysiological recordings in the LA during emotional learning indicate neural plasticity (Quirk et al., 1995; Collins and Pare, 2000; Maren, 2000; Pare and Collins, 2000). Our findings demonstrate that electrolytic and, more importantly, excitotoxic lesions confined to the LA can also substantially attenuate retention of contextually conditioned fear. Previous studies have demonstrated that both post-shock freezing and freezing in the retention test of the simple contextual fear conditioning paradigm used in the present study are associatively conditioned (Faneslow, 1990). Although some types of contextual fear conditioning may be hippocampal-dependent (Kim and Fanselow, 1992; Phillips and LeDoux, 1992), it appears that learning in the simple contextual conditioning paradigm is not (Phillips and LeDoux, 1994; Fanselow, 2000). Nonetheless, the present study demonstrates that the LA is not just important in cue-specific fear conditioning (LeDoux, 2000) but also for conditioning in a paradigm lacking a specific cue.

The present study further indicates that LA neurons are not necessary for post-shock freezing indicative of short-term memory but are important for long-term memory of fear conditioning. The decrease in freezing during both the post-shock and retention test periods after electrolytic lesions confounds the interpretation of the involvement of the LA in learning and memory of fear, which could be viewed as a performance deficit (Cahill et al., 1999). However, NMDA lesions clarify that cells of the LA are not necessary for short-term memory or freezing behavior per se but are important for normal levels of long-term memory of fear. Sparing of normal levels of post-shock freezing but substantially reducing retention test freezing with LA excitotoxic lesions indicates that the LA is necessary for long-term but not short-term memory of conditioned fear.

The effects on fear-conditioned freezing were different when neural damage also included the BA and ASTR. Freezing was severely attenuated in both the post-shock period and in the retention test by larger NMDA lesions that damaged the LA, ASTR, and BA. Several studies have now demonstrated that post-shock freezing as well as freezing in a retention test of contextual fear are significantly diminished in rats with large excitotoxic lesions of the BLC (Maren, 1999b; Cahill et al., 2000). The present study indicates there is a difference in the role of the LA and BA in short-term and long-term memory of fear conditioning.

Although the present study can dissociate the effects of LA lesions on short-term memory (no effect on post-shock freezing) from long-term memory (a reduction in freezing on the retention test), it does not discriminate between consolidation and retrieval processes. Other data suggest that the LA may be involved primarily in late phases (consolidation) of learning and long-term potentiation. Although some studies have found an initial increase and then decrease in activity in LA neurons during conditioning (Quirk et al., 1995, 1997), several groups have demonstrated increased cellular activity in LA neurons commensurate with classical fear conditioning in cats and rats (Collins and Pare, 2000; Maren, 2000; Pare and Collins, 2000). Electrophysiological recording during discriminatory instrumental fear conditioning in rabbits demonstrates that LA cells respond robustly during late phases of learning but not earlier phases (Gabriel and Talk, 2001). In contrast, BA cells respond robustly during early phases and diminish as learning proceeds (Gabriel and Talk, 2001), possibly indicating that the BA is more important for short-term than long-term memory. The LA also shows a late, persistent phase of LTP that is dependent on protein kinase A and mitogenactivated protein kinase (Huang et al., 2000). Interestingly, the expression of one of the targets of these kinases, the immediateearly gene egr-1, is increased specifically within the LA after contextual fear conditioning (Rosen et al., 1998; Malkani and Rosen, 2000), suggesting part of a molecular pathway in the LA for long-term memory processes.

\section{Role of the LA in unconditioned freezing to a predatory odor}

Previous research has demonstrated that rats will freeze, avoid, and have physiological responses to predator odors (including TMT) that are indicative of unconditioned fear (Cattarelli and Chanel, 1979; Blanchard and Blanchard, 1989; Heale et al., 1994; Berton et al., 1998; Burwash et al., 1998; Perrot-Sinal et al., 1999; 
Morrow et al., 2000b; Wallace and Rosen, 2000). Because large lesions of the entire amygdala blocked fear responses to the presentation of a cat (Blanchard and Blanchard, 1972; Fox and Sorenson, 1994), we expected that amygdala lesions would also reduce freezing to TMT. Indeed, electrolytic lesions of the LA significantly reduced freezing to TMT, and larger LA/ASTR lesions almost totally abolished freezing. In contrast, NMDA lesions of the LA had no effect on freezing to TMT, whereas larger lesions of the $\mathrm{LA}+$ group produced only a slight reduction in freezing to TMT. The data suggest that fibers passing through the basolateral complex and ASTR, and not the cells of the LA, ASTR, and BA, are responsible for the large reductions in freezing. Although it is possible that a larger reduction in unconditioned freezing would occur with larger NMDA lesions, the strong effects of these lesions on contextually conditioned freezing compared with the weak effects on freezing to TMT suggest that conditioned and unconditioned freezing to TMT rely on different amygdala circuitry.

It may not be surprising that the NMDA lesions of the LA did not affect freezing to TMT. The main olfactory bulb or olfactory cortex does not project directly to the LA. Nevertheless, olfactory information can enter the amygdala via the piriform cortex and superficial amygdaloid nuclei, which project to the insular cortex and then through insular efferents to the central nucleus of the amygdala (Shipley et al., 1995). TMT may also activate the accessory olfactory bulb via the vomeronasal organ and enter the amygdala through a direct projection to the medial nucleus of the amygdala (Shipley et al., 1995). However, what is of interest from the results of the present study is that excitotoxic lesions produced profound effects on conditioned freezing but had minimal effects on unconditioned freezing to a predator odor, again strongly indicating that LA and BA are not necessary for production of freezing behavior per se. Although these findings are exciting, more work needs to determine whether the results are unique to TMT and unconditioned fear inducing olfactory stimuli. Indeed, lesions of the BLC or LA only disrupt unconditioned fear responses to a brightly lit environment (Walker and Davis, 1997), a loud auditory stimulus (Bellgowan and Helmstetter, 1996), and a large ball of cat fur (A. Vazdarjanova, personal communication). Furthermore, the BLC appears to be necessary for fear conditioning when an olfactory stimulus is paired with foot shock (Otto et al., 2000). These results suggest that the BLC may process visual and auditory stimuli involved in unconditioned fear responses and may be necessary for olfactory fear conditioning as it is with other types of fear conditioning.

In summary, the role of the LA in fear conditioning appears unique to long-term memory processes because freezing immediately after learning was unaffected by NMDA lesions, but long-term memory was reduced. Furthermore, an interesting dissociation between the effects of lesions of nuclei of the basolateral complex on conditioned and unconditioned fear suggests that these amygdaloid nuclei are important for learning and memory of fear but not for expression of some types of unconditioned fear.

\section{REFERENCES}

Bellgowan PS, Helmstetter FJ (1996) Neural systems for the expression of hypoalgesia during nonassociative fear. Behav Neurosci 110:727-736.

Berton F, Vogel E, Belzung C (1998) Modulation of mice anxiety in response to cat odor as a consequence of predators diet. Physiol Behav 65:247-254.

Blanchard DC, Blanchard RJ (1969) Crouching as an index of fear. J Comp Physiol Psychol 67:370-375.

Blanchard DC, Blanchard RJ (1972) Innate and conditioned reactions to threat in rats with amygdaloid lesions. J Comp Physiol Psychol 81:281-290.

Blanchard RJ, Blanchard DC (1989) Antipredator defensive behaviors in a visible burrow system. J Comp Psychol 103:70-82.

Burwash MD, Tobin ME, Woolhouse AD, Sullivan TP (1998) Laboratory evaluation of predator odors for eliciting an avoidance response in roof rats. J Chem Ecol 24:49-66.

Cahill L, Weinberger NM, Roozendaal B, McGaugh JL (1999) Is the amygdala a locus of "conditioned fear"? Neuron 23:227-228.

Cahill L, Vazdarjanova A, Setlow B (2000) The basolateral amygdala complex is involved with, but not necessary for, rapid acquisition of Pavlovian "fear conditioning." Eur J Neurosci 12:3044-3050.

Campeau S, Davis M (1995) Involvement of the central and basolateral complex of the amygdala in fear conditioning measured with fearpotentiated startle in rats trained concurrently with auditory and visual conditioned stimuli. J Neurosci 15:2301-2311.

Campeau S, Miserendino MJ, Davis M (1992) Intra-amygdala infusion of the $N$-methyl-D-aspartate receptor antagonist AP5 blocks acquisition but not expression of fear-potentiated startle to an auditory conditioned stimulus. Behav Neurosci 106:469-574.

Cattarelli M, Chanel J (1979) Influence of some biologically meaningful odorants on the vigilance states of the rat. Physiol Behav 23:831-838.

Collins DR, Pare D (2000) Differential fear conditioning induces reciprocal changes in the sensory response of lateral amygdala neurons to the CS $(+)$ and CS $(-)$. Learn Mem 7:97-103.

Cousens G, Otto T (1998) Both pre- and posttraining excitotoxic lesions of the basolateral amygdala abolish the expression of olfactory and contextual fear conditioning. Behav Neurosci 112:1092-1103.

Davis M (1997) Neurobiology of fear responses: the role of the amygdala. J Neuropsychiatry Clin Neurosci 9:382-402.

Faneslow MS (1990) Factors governing one trial contextual conditioning. Anim Learn Behav 18:264-270.

Fanselow MS (2000) Contextual fear, gestalt memories, and the hippocampus. Behav Brain Res 110:73-81.

Fanselow M, LeDoux JE (1999) Why we think plasticity underlying Pavolvian fear conditioning occurs in the basolateral amygdala. Neuron 23:229-232.

Fox RJ, Sorenson CA (1994) Bilateral lesions of the amygdala attenuate analgesia induced by diverse environmental challenges. Brain Res 648:215-221.

Gabriel M, Talk A (2001) A tale of two paradigms: lessons learned from parallel studies of discriminative instrumental learning and classical eyeblink conditioning. In: Feschrift volume for Professor Richard F. Thompson (Steinmetz J, Solomon P, eds). Mahwah, NJ: Erlbaum, in press.

Heale VR, Vanderwolf CH, Kavaliers M (1994) Components of weasel and fox odors elicit fast wave bursts in the dentate gyrus of rats. Behav Brain Res 63:159-165.

Hotsenpillar G, Williams JL (1997) A synthetic predator odor (TMT) enhances conditioned analgesia and fear when paired with a benzodiazepine receptor inverse agonist (FG-7142). Psychobiology 25:83-88.

Huang Y-Y, Martin K, Kandel ER (2000) Both protein kinase A and mitogen-activated protein kinase are required in the amygdala for the macromolecular synthesis-dependent late phase of long-term potentiation. J Neurosci 20:6317-6325.

Kim JJ, Fanselow MS (1992) Modality-specific retrograde amnesia of fear. Science 256:675-677.

Kim JJ, Rison RA, Fanselow MS (1993) Effects of amygdala, hippocampus, and periaqueductal gray lesions on short- and long-term contextual fear. Behav Neurosci 107:1093-1098.

LeDoux JE (2000) Emotion circuits in the brain. Annu Rev Neurosci 23:155-184.

LeDoux JE, Cicchetti P, Xagoraris A, Romanski LM (1990a) The lateral amygdaloid nucleus: sensory interface of the amygdala in fear conditioning. J Neurosci 10:1043-1054.

LeDoux JE, Farb CR, Ruggiero DA (1990b) Topographic organization of neurons in the acoustic thalamus that project to the amygdala. J Neurosci 10:1043-1054.

Malkani S, Rosen JB (2000) Specific induction of immediate early growth response gene 1 (EGR-1) in the lateral nucleus of the amygdala following contextual fear conditioning in rats. Neuroscience 97:693-702.

Maren S (1999a) Long-term potentiation in the amygdala: a mechanism of emotional learning and memory. Trends Neurosci 22:561-567.

Maren S (1999b) Neurotoxic basolateral amygdala lesions impair learning and memory but not the performance of conditional fear in rats. J Neurosci 19:8696-8703.

Maren S (2000) Auditory fear conditioning increases CS-elicited spike firing in lateral amygdala neurons even after extensive overtraining. Eur J Neurosci 12:4047-4054.

Maren S, Aharonov G, Fanselow MS (1996a) Retrograde abolition of conditional fear after excitotoxic lesions in the basolateral amygdala of rats: absence of a temporal gradient. Behav Neurosci 110:718-726.

Maren S, Aharonov G, Stote DL, Fanselow MS (1996b) N-Methyl-Daspartate receptors in the basolateral amygdala are required for both 
acquisition and expression of conditional fear in rats. Behav Neurosci 110:1365-1374.

McDonald AJ (1998) Cortical pathways tot he mammalian amygdala. Prog Neurobiol 55:257-332.

Miserendino MJD, Sananes CB, Melia KR, Davis M (1990) Blocking of acquisition but not expression of conditioned fear-potentiated startle by NMDA antagonists in the amygdala. Nature 345:716-718.

Morrow BA, Redmond AJ, Roth TH, Elsworth JD (2000a) The predator odor, TMT, displays a unique, stress-like pattern of dopaminergic and endocrinological activation in the rat. Brain Res 864:146-151.

Morrow BA, Roth RH, Elsworth JD (2000b) TMT, a predator odor, elevates mesoprefrontal dopamine metabolic activity and disrupts short-term working memory in the rat. Brain Res Bull 52:519-523.

Muller J, Coordimas KP, Fridel Z, LeDoux JE (1997) Functional inactivation of the lateral and basal nuclei of the amygdala by muscimol infusion prevents fear conditioning to an explicit conditioned stimulus and to contextual stimuli. Behav Neurosci 111:683-691.

Otto T, Cousens G, Herzog C (2000) Behavioral and neuropsychological foundations of olfactory fear conditioning. Behav Brain Res 110:119-128.

Pare D, Collins DR (2000) Neuronal correlates of fear in the lateral amygdala: multiple extracellular recordings in conscious cats. J Neurosci 20:2701-2710.

Paxinos G, Watson C (1998) The rat brain in stereotaxic coordinates, Ed 4. San Diego: Academic.

Perrot-Sinal T, Ossenkopp K, Kavaliers M (1999) Brief predator odor exposure activates the HPA axis independent of locomotor changes. NeuroReport 10:775-780.

Phillips RG, LeDoux JE (1992) Differential contribution of amygdala and hippocampus to cued and contextual fear conditioning. Behav Neurosci 106:274-285.

Phillips RG, LeDoux JE (1994) Lesions of the dorsal hippocampal formation interfere with background but not foreground contextual fear conditioning. Learn Mem 1:34-44.

Pitkanen A, Savander V, LeDoux JE (1997) Organization of intraamygdaloid circuitries in the rat: an emerging framework for understanding functions of the amygdala. Trends Neurosci 20:517-523.

Quirk GJ, Repa C, LeDoux JE (1995) Fear conditioning enhances short latency auditory responses of lateral amygdala neurons: parallel recordings in the freely moving rat. Neuron 15:1029-1039.
Quirk GJ, Armony JL, LeDoux JE (1997) Fear conditioning enhances different temporal components of tone-evoked spike trains in auditory cortex and lateral amygdala. Neuron 19:613-624.

Rogan MT, Staubli UV, LeDoux JE (1997) Fear conditioning induces associative long-term potentiation in the amygdala. Nature 390:604-607.

Rosen JB, Fanselow MS, Young SL, Sitcoske M, Maren S (1998) Immediate-early gene expression in the amygdala following footshock stress and contextual fear conditioning. Brain Res 796:132-142.

Shi CJ, Cassell MD (1999) Perirhinal cortex projections to the amygdaloid complex and hippocampal formation in the rat. J Comp Neurol 406:299-328.

Shipley MT, McLean JH, Ennis M (1995) Olfactory system. In: The rat nervous system, Ed 2 (Paxinos G, ed). San Diego: Academic.

Swanson LW, Petrovich GD (1998) What is the amygdala? Trends Neurosci 21:323-331.

Thompson BL, Rosen JB (2000) The effects of TRH on acoustic startle, conditioned fear, and active avoidance in rats. Neuropeptides $34: 38-44$.

Vazdarjanova A (2000) Does the basolateral amygdala store memories for emotional events? Trends Neurosci 23:345-346.

Vernet-Maury E, Polak EH, Demael A (1984) Structure-activity relationship of stress-inducing odorants in the rat. J Chem Ecol 10:1007-1018.

Vernet-Maury E, Constant B, Chanel J (1992) Repellent effects of trimethylthiazoline in the wild rat Rattus novegicus berkenout. In: Chemical signals in vertebrates (Doty RL, Muller-Schwarze D, eds), pp 305-310. New York: Plenum.

Walker DL, Davis M (1997) Double dissociation between the involvement of the bed nucleus of the stria terminalis and the central nucleus of the amygdala in startle increases produced by conditioned versus unconditioned fear. J Neurosci 17:9375-9383.

Wallace KJ, Rosen JR (2000) Predator odor as an unconditioned fear stimulus in rats: elicitation of freezing by trimethylthiazoline, a component of fox feces. Behav Neurosci 114:912-922.

Wilensky AE, Schafe GE, LeCoux JE (2000) The amygdala modulates memory consolidation of fear-motivated inhibitory avoidance learning but not classical fear conditioning. J Neurosci 20:7059-7066. 